\title{
Naturheilkunde oder Komplementärmedizin?
}

Die Vielfalt der Wahrheitsansprüche unter denjenigen Behandlungsmethoden, die versprechen, etwas für die menschliche $\mathrm{Ge}$ sundheit tun zu können, ist etwas verwirrend. Ich empfinde dies sowohl für den Gegensatz zwischen der sogenannten Schulmedizin und den naturheilkundlichen Verfahren als auch für diese letzteren selbst. Was haben beispielsweise Phytotherapie, Akupunktur, Hydrotherapie, Bewegungstherapie, Neuraltherapie, Manuelle Therapie, Ableitende Verfahren, Reizkörpertherapie, Homöopathie, Blütentherapie, Sauerstofftherapie, Irisdiagnostik und Anthroposophische Medizin miteinander gemein, das sie alle gleichermassen als Naturheilkunde zu verstehen erlaubt? Ich habe darauf bisher keine überzeugende Antwort gefunden und kann Malte Bührings Frage aus dem Editorial des Hefts 3/1999 dieser Zeitschrift, ob Komplementärmedizin eigentlich Naturheilkunde sei oder umgekehrt, deshalb einstweilen nur in diesem erweiterten Feld aufnehmen.

\section{Naturheilkunde und «Schulmedizin»}

Was den Gegensatz zur «Schulmedizin» angeht, so leuchtet es mir vor allem nicht ein, wie ein Arzt auf den Anspruch verzichten kann, dass alles, was er tut, auf Naturheilkunde beruht. Denn jedes ärztliche Handeln setzt voraus, der Natur des Menschen - vor allem in körperlicher Hinsicht - im Ganzen der Natur so weit kundig zu sein, dass man eine Krankheit heilen oder die Selbstheilungskräfte des Kranken stärken kann. Dies gilt auch für diejenigen Ärzte, die der Natur des Menschen im Ganzen der Natur in einer naturwissenschaftlich fundierten Weise kundig sind. Wieso aber gilt oder versteht sich dann nicht auch die «Schulmedizin» als Naturheilkunde?

Eigentlich ist die sogenannte Schulmedizin nur derjenige Teil der Naturheilkunde, dem die klassischen Naturwissenschaften externe Begründungen für das ärztliche Handeln geliefert haben. Warum aber sollte dieses Handeln ausgerechnet deshalb, weil ihm die Wissenschaften von der Natur zu Hilfe gekommen sind, der Natur des Menschen weniger kundig sein? Wie es dazu gekommen ist, einen Teil der Heilberufe als «Schulmedizin» aus dem allgemeinen Kor- pus der Naturheilkunde auszugrenzen, ist historisch nachvollziehbar. Geltungsbedürfnisse und soziale Differenzierungen innerhalb der Heilberufe haben dabei aber möglicherweise doch eine wesentlich grössere Rolle gespielt, als es dem Selbstverständnis der ärztlichen Kunst sachlich angemessen gewesen wäre.

Man weiss ja auch gar nicht, welche Heilmethoden, die jetzt noch als naturkundlich gelten, demnächst ebenfalls naturwissenschaftlich abgesegnet werden und dann in das andere Lager übergehen. Die Auseinandersetzung erinnert insoweit an diejenige zwischen Naturwissenschaftlern und Theologen zu der Zeit, als die letzteren ihren Kompetenzbereich so abgrenzten, dass sie für gerade das zuständig sein wollten, was naturwissenschaftlich unerklärbar sei. Diese Arbeitsteilung ist den Theologen nicht gut bekommen, denn die Naturwissenschaften waren so expansiv, dass der Theologie schließlich in eigener Zuständigkeit nichts mehr übrig blieb. Der Grund für die scheinbare Überflüssigkeit der Theologie war aber nur ein falsches Selbstverständnis. Ob die derzeitige Arbeitsteilung zwischen Naturheilkunde und «Schulmedizin» genauso falsch ist, weiss ich nicht.

Ich vermute, dass wir noch nicht gut genug verstanden haben, was eigentlich das Proprium der Naturheilkunde ist, und dass es der «Schulmedizin» gut tun würde, in einem neuen Sinn Naturheilkunde zu werden. In diesem Sinn nehme ich im folgenden den Gedanken der Komplementarität auf, der dieser Zeitschrift den Namen gegeben hat. Meine Überlegungen entspringen aber nicht nur einem Bedürfnis nach philosophischer Klarheit, sondern sind auch gesundheitspolitisch motiviert.

Die heutige Arbeitsteilung im Gesundheitswesen unterscheidet im Grunde nur Kompetenzen, entspricht aber eigentlich weder den Bedürfnissen der Gesunden noch denen der Kranken und ist insoweit dysfunktional. Denn die «Schulmedizin» einerseits ist ein weitgehend erfolgreiches Instrumentarium zur Heilung akuter und schwerer Krankheiten, versagt aber bei Alltagsbeschwerden (Erkältungen, Rückenschmerzen etc.), chronischen Erkrankungen, Somatisierungen psychischer Konflikte und in der Vor- bzw. Nachsorge. Die naturheilkundlichen Verfahren andererseits sind bei all ihrer Heterogenität gerade dort stark, wo die herrschende Medizin schwach ist, und vor allem darauf beruht ihr zunehmendes Anse-

\section{KARGER}

Fax +497614520714

E-mail kargergmbh@aol.com

www.karger.com (c) 1999 S. Karger GmbH, Freiburg

Accessible online at:

http://BioMedNet.com/karger 
hen. In der Finanzkrise des Gesundheitswesens kommt hinzu, dass viel Geld gespart werden könnte, wenn die naturheilkundliche Prävention dazu führen würde, dass weniger akute und schwere Krankheiten auftreten.

Ist es aber eine gute Arbeitsteilung, wenn die einen sozusagen fürs Grobe und die andern für die Feinheiten zuständig sind? Soviel ich sehe, gibt es in den Übergangsbereichen Verwerfungen, welche den Patienten nicht gut tun. Dies gilt wohl vor allem für den Übergang in die Nachsorge, wenn eine schwere Krankheit nicht mehr akut ist. Es liegt aber auch begrifflich auf der Hand, dass es nicht gut sein kann, wenn die einen im wesentlichen für die Gesunden und Kränkelnden, die andern für die richtig Kranken zuständig sind (soweit ihnen naturwissenschaftlich zu helfen ist). Denn die Aufgabe des Arztes liegt vor allem in dem «Zwischen», das in den blinden Fleck der gegenwärtigen Arbeitsteilung fällt.

Meinem Eindruck nach ist also die Unterscheidung zwischen Naturheilkunde und «Schulmedizin» bisher

- begrifflich schwach, weil sie sich nicht aus den Aufgaben, sondern aus einer externen Entwicklung, derjenigen der Naturwissenschaften, ergeben hat;

- praktisch stark durch die ständischen Abgrenzungen von Kompetenzen, jedoch so, dass diese Unterscheidung nicht im Interesse der Gesunden und der Kranken ist.

Damit kann man eigentlich nicht zufrieden sein. In dieser Situation nun scheint mir ein gütiges Geschick der Naturheilkunde den Gedanken der Komplementärmedizin beschert zu haben. In diese Richtung deuteten auch Malte Bührings Überlegungen im zweiten Teil seines Beitrags.

\section{Gemeinsame Perspektive: Eine komplementäre Naturheilkunde}

Ich gehe hier nicht darauf ein, woher der Begriff «Komplementarität» in der Medizin kommt und wie er ursprünglich gemeint war, sondern nehme den Gedanken in seinem naturphilosophischen Sinn auf. Er stammt von Niels Bohr, dem dänischen Philosophen und Physiker, und ist von ihm ursprünglich im Rahmen der «Kopenhagener Interpretation» der Quantentheorie eingeführt worden (1927). Komplementäre Verhältnisse für eine Spezialität der Wissenschaftstheorie zu halten wäre jedoch ein grosser Irrtum. Komplementarität ist vielmehr der Schlüsselbegriff für den Übergang von der klassischen Physik zur Quantentheorie, d. h. von einer technisch zwar noch begrenzt richtigen, in ihrem erkenntnisleitenden Natur- und Menschenbild aber ganz unzulänglichen Physik zu einer Wissenschaft von der Naturzugehörigkeit des Menschen.

Das Interessante für die Medizin und insbesondere für die Naturheilkunde ist dabei, dass die sogenannte Schulmedizin sich auf die klassische, d.h. vor-quantentheoretische und insoweit überholte Naturwissenschaft stützt, sich also die Frage gefallen lassen muss, warum sie noch immer keine komplementäre Wissenschaft ist. Die Naturheilkunde steht zwar vor derselben Frage, stützt sich aber doch nicht grundsätzlich auf eine jedenfalls nur beschränkt gültige
Art von Antworten. Dabei kommt es für die Medizin vor allem darauf an, dass die klassische Naturwissenschaft in ihrem Naturverständnis definitiv überholt ist. Was wir unter der Natur verstehen, ist aber immer eng verschränkt mit unserem menschlichen Selbstverständnis.

Philosophisch war der Schritt von der klassischen Naturwissenschaft zur Quantentheorie also der Übergang zu einem neuen Menschen- und Naturbild. Daran sollte die Medizin eigentlich nicht vorbeigehen, zumal das Natur- und Menschenbild der «Schulmedizin» aus der ärztlichen Praxis weitreichenden und meines Erachtens berechtigten Zweifeln ausgesetzt ist. Ich schliesse mit einigen Andeutungen, in welcher Hinsicht sowohl die «Schulmedizin» als auch die Naturheilkunde durch das Natur- und Menschenbild der Komplementaritäts-Philosophie herausgefordert sind und welche Chancen darin liegen. Den Grundgedanken veranschaulicht eine kluge Kinderfrage.

Als der Vater seiner kleinen Tochter die Photographien zeigte, die er auf einem gemeinsamen Spaziergang aufgenommen hatte, fand sie sich darauf ohne ihn und fragte verwundert: Aber Papa, wo bist du denn? Er war doch bei ihr gewesen, und das war eigentlich sogar die Hauptsache. Ausserdem hatte er selbst die Bilder gemacht, gehörte also dazu. Wieso war er auf den Bildern nicht zu sehen?

Geradeso wie dem Kind mit den Photographien ergeht es uns mit der klassischen Naturwissenschaft. Auf dem Bild, das sie von der Welt entwirft, kommen wir selber nicht vor. Wir stehen immer nur dahinter. Es ist so, als sähen wir alles wie unter einer Tarnkappe, also ohne selbst gesehen zu werden, oder einfach von oben her, so wie ein interplanetarisches Eroberervolk sich die Erde von aussen ansehen würde. Das in dieser Wissenschaft erkenntnisleitende Natur- und Menschenbild ist also offensichtlich falsch, denn wir sind keine interplanetarischen Eroberer, sondern Erdensöhne und Erdentöchter, aus der Naturgeschichte hervorgegangen. In uns kommt die Natur zur Sprache, aber wir sind selbst ein Teil der Natur, und sie «treibt sich mit uns fort», wie Goethe sagte.

Man kann aus der klassischen Naturwissenschaft nicht nur die Ergebnisse übernehmen, sondern dazu gehört immer auch die Haltung, unter der diese ihren Sinn haben. Deshalb objektiviert der klassisch naturwissenschaftliche Mediziner den Patienten genauso von oben her wie sein Vorbild, oder als gehörte er selbst nicht dazu. Ein Gegenbild ist der «Gestaltkreis» Viktor von Weizsäckers, in den der Arzt und der Patient gemeinsam eintreten, so dass auch der Arzt aus einer Behandlung als ein anderer hervorgeht. Eine mitverantwortliche Beteiligung statt der bloss «gesund machenden» Behandlung wird mittlerweile auch von vielen Patienten gewünscht.

Die komplementäre Naturerfahrung unterscheidet sich dadurch von der klassischen, dass die Umstände, unter denen etwas erfahren wird, grundsätzlich zur Wirklichkeit des Erfahrenen gehören. Philosophisch handelt es sich dabei um einen sehr grundsätzlichen Pragmatismus in dem Sinn, dass die Dinge sich uns immer nur so zeigen, wie wir es mit ihnen zu tun haben. Wir beschreiben die Welt hier nicht mehr so, als gehörten wir nicht dazu, sondern wir 
gehören dazu und der Gegenstand auch. Wir beziehen uns auf ihn in einer bestimmten Weise des Mitseins. Es gibt aber verschiedene Weisen des Mitseins, und manche schliessen einander aus. Man kann es dann nicht gleichzeitig auf diese oder jene Weise mit dem Gegenstand zu tun haben, und doch widersprechen sich die einander ausschließenden Erfahrungen nicht, sondern sie sind komplementär, d. h., sie ergänzen einander zu einem Ganzen.

Für die Naturheilkunde wie für die «Schulmedizin» ist die Komplementarität ein Programm, aus dem sie beide nicht unverändert hervorgehen würden. Nichts aber ist so verbindend wie ein gemeinsamer Weg. Ein beiderseits veränderter Umgang mit dem Gesunden und Kranken könnte dann vielleicht eine Komplementäre Naturheilkunde heissen. Wenn dieser Weg durch Beiträge und durch eine lebendige Diskussion unter den Lesern hier schon eingeschlagen würde, trüge diese Zeitschrift ihren Namen ganz gewiss zu Recht.

Klaus Michael Meyer-Abich, Essen

\section{Literatur}

Die in diesem Editorial teilweise nur angedeuteten Gedanken sind ausführlicher entwickelt in Klaus Michael Meyer-Abich, Gerhard Scherhorn et al.: Vom Baum der Erkenntnis zum Baum des Lebens - Ganzheitliches Denken der Natur in Wissenschaft und Wirtschaft (München, C. H. Beck, 1997). Dort finden sich auch weiterführende Literaturangaben. 